\title{
Prevalência de inatividade física na adolescência: revisão sistemática
}

A adolescência é uma fase importante no que se diz respeito a pratica de atividade física, que por sua vez está relacionada à proteção de futuras patologias promoção e manutenção da saúde. A presente pesquisa buscou descrever as características metodológicas dos estudos selecionados e identificar a prevalência de inatividade física de adolescentes escolares. O estudo em questão se trata de uma revisão sistemática, formulada através de manuscritos científicos em formato de artigo. Buscaram-se artigos pertinentes a temática em duas bases de dados eletrônicos: Lilacs e SciELO, usando-se os descritores: comportamento sedentário exercício e adolescente, combinados com o operador booleano 'and'. Foram utilizados 13 artigos após critérios de inclusão e exclusão. Os dados gerais de prevalência de inatividade física variaram entre $19,7 \%$ a $81,6 \%$. Quando compilados de acordo com o sexo, os estudos revelam elevada prevalência de inatividade em ambos os sexos, atingidos picos de $86 \%$ em mulheres e $81,6 \%$ em homens. Conclui-se que os adolescentes têm elevada prevalência de inatividade física, fato esse evidenciado em ambos os sexos.

Palavras-chave: Comportamento sedentário; Exercício; Adolescente.

\section{Prevalence of physical inactivity in adolescence: systematic review}

Adolescence is an important phase with regard to the practice of physical activity, which in turn is related to protecting future pathologies, promoting and maintaining health. This research sought to describe the methodological characteristics of the selected studies and to identify the prevalence of physical inactivity among school adolescents. The study in question is a systematic review, relevant to the theme were searched for in two electronic databases: Lilacs and SciELO, using the descriptors: sedentary behavior, exercise and adolescent, combined with the Boolean operator 'and'. 13 articles were used after inclusion and exclusion criteria. The general data on the prevalence of physical inactivity ranged from $19.7 \%$ to $81.6 \%$. When compiled according to gender, studies show a high prevalence of inactivity in both sexes, reaching peaks of $86 \%$ in women and $81.6 \%$ in men. It is concluded that adolescents have a high prevalence of physical inactivity, a fact evidenced in both genders.

Keywords: Sedentary behavior; Exercise; Adolescent.

Topic: Educação Física

Reviewed anonymously in the process of blind peer.
Received: 11/09/2019

Approved: 06/01/2020
Marcos Antonio Araújo Bezerra (D)

Centro Universitário Dr. Leão Sampaio, Brasil

http://lattes.cnpq.br/4643352879633283

http://orcid.org/0000-0002-3385-4024

marcosbezerra@univs.edu.br

José Suesley Ribeiro Sousa (iD)

Centro Universitário Dr. Leão Sampaio, Brasil

http://lattes.cnpq.br/175968030174782

http://orcid.org/0000-0002-9686-3542

swesleyribeiro@hotmail.com

José de Caldas Simões Neto (ib)

Centro Universitário Dr. Leão Sampaio, Brasil

http://lattes.cnpq.br/0470733825644726

http://orcid.org/0000-0003-1036-2315

josecaldas@leaosampaio.edu.br
Antoniel dos Santos Gomes Filho (id

Centro Universitário Vale do salgado, Brasil

http://lattes.cnpq.br/9563145614494252

http://orcid.org/0000-0003-2230-4315

antoniel.historiacomparada@gmail.com

Lara Belmudes Bottcher (it)

Centro Universitário Dr. Leão Sampaio, Brasil

http://lattes.cnpq.br/4112151773664370

http://orcid.org/0000-0002-5504-4102

larabottcher@leaosampaio.edu.b
Referencing this:

BEZERRA, M. A. A.; SOUSA, J. S. R.; SIMÕES NETO, J. C.; GOMES FILHO, A. S.; BOTTCHER, L. B.. Prevalência de inatividade física na adolescência: revisão sistemática. Health of Humans, v.2, n.1, p.1-9, 2019. DOI: http://doi.org/10.6008/CBPC2674-6506.2020.001.0001

DOI: 10.6008/CBPC2674-6506.2020.001.0001 


\section{INTRODUÇÃO}

A adolescência é uma fase importante no que se diz respeito a pratica de alguma atividade física, sendo que nessas fases estes passam por muitas mudanças, e a pratica de atividade física, pode auxiliar em torno de tudo que acontece no seu corpo e mente, o tornando uma pessoa ativa, protegendo de futuras patologias, promovendo uma manutenção da saúde, e por consequência prolongando esse comportamento por sua vida adulta (SILVA et al., 2018).

A OMS (2018) aponta que no ranking dos principais fatores de risco da mortalidade mundial, a inatividade física ocupa o quarto lugar, sendo responsável por cerca de 3,2 milhões de morte anual. Estimase que pessoas fisicamente inativas têm risco de mortalidade em torno de 20 a 30\%, quando confrontadas as pessoas consideradas fisicamente ativas.

No período de 2009 a 2015 segundo os dados do IBGE (Instituto Brasileiro de Geografia e Estatística) evidenciaram a diminuição da prática de atividade física, concentrada em 300 minutos semanal. Ressalta-se também uma prevalência elevada de inatividade física global (OLIVEIRA et al., 2017). Warburton et al. (2017) propõem que atividade física apresenta diversas vantagens ao corpo humano, tanto na prevenção de doenças como no tratamento e reabilitação dessas patologias, quando praticada na infância, ainda apresenta outro grande benefício, tende a se delongar durante a vida adulta.

Alberto et al. (2015) apontaram que índices de inatividade física em adolescentes escolares haviam tido elevado crescimento, onde se pode relatar que alguns fatores estão relacionados a esse crescimento, tais como: fatores demográficos/socioeconômicos, idade e sexo, citando também as questões de prevenção às DCNT (doenças crônicas não transmissíveis) e aos benefícios a saúde.

Segundo Marques et al. (2015) na adolescência a prevenção de DCNTs está ligada diretamente a prática de atividade física, onde a mesma pode influenciar na regulação da pressão arterial, reforço da estrutura óssea, além de prolongar esse comportamento ativo na vida adulta. Ainda relata que em relação à atividade física, é na adolescência o período considerado de maior importância, destacando, a necessidade de oportunidades e estímulos para que esses níveis de atividade física estejam cada vez mais adequados entre os jovens, assim se verificou a carência de realização de algumas políticas públicas em busca da redução das questões em torno da inatividade física dos adolescentes. Assim, o objetivo da presente pesquisa foi descrever as características metodológicas dos estudos selecionados e identificar a prevalência de inatividade física de adolescentes escolares.

\section{METODOLOGIA}

O estudo em questão se trata de uma revisão sistemática, formulada através de manuscritos científicos em formato de artigo. Buscaram-se artigos pertinentes a temática em duas bases de dados eletrônicos: Lilacs e SciELO, usando-se os descritores: comportamento sedentário, exercício e adolescente, combinados com o operador booleano 'and'. Ressalta-se que o objetivo do estudo foi verificar a prevalência de inatividade física em adolescente, onde o descritor 'Comportamento sedentário' foi utilizado em 
substituição ao termo 'inatividade física', já que este não se encaixa como um descritor nos bancos de dados eletrônicos para se encontrar estudos onde há prevalência de inatividade física.

Tabela 1: Descrição das bases de dados, descritores, operador utilizados.

\begin{tabular}{|l|l|l|l|l|}
\hline Base & Descritor & Op. & Descritor & Quantidade de artigos \\
\hline \multirow{4}{*}{ Scielo } & Comportamento sedentário & AND & Exercício & 20 \\
\cline { 2 - 5 } & Exercício & AND & Adolescente & 173 \\
\cline { 2 - 5 } & Adolescente & AND & Comportamento sedentário & 54 \\
\hline \multirow{5}{*}{ Lilacs } & Comportamento sedentário & AND & Exercício & 197 \\
\cline { 2 - 5 } & Exercício & AND & Adolescente & 1331 \\
\cline { 2 - 5 } & Adolescente & AND & Comportamento sedentário & 242 \\
\hline
\end{tabular}

Logo em seguidas foram aplicados os seguintes critérios de inclusão: artigos originais e disponíveis na integra, entre os anos de 2015 a 2019, no idioma português e artigos observacionais citáveis desenvolvidos em território nacional. Foram excluídos artigos de revisão sistemática e bibliográfica, estudos duplicados, estudos que não estejam relacionados ao tema principal da pesquisa e cujo objetivo da pesquisa não foi identificar as prevalências de inatividade física em adolescentes.

Foram encontrados a partir das bases de dados eletrônicas ( $n=2017)$ artigos, sendo ( $n=247)$ artigos na plataforma SCIELO, e $(n=1.770)$ artigos na plataforma LILACS. Quando aplicados os filtros (critérios de inclusão) restaram 307 artigos, por fim foram excluídos 294 artigos de acordo com os critérios de exclusão, totalizando assim 13 artigos como amostra final da presente pesquisa. (Fluxograma 1).

\begin{tabular}{|c|c|}
\hline \multicolumn{2}{|c|}{ IDENTIFICAÇÃO } \\
\hline $\begin{array}{l}\text { Registros identificados através das bases de dados } \\
\text { (Scielo) } \\
\underline{\mathbf{2 4 7} \text { artigos }}\end{array}$ & $\begin{array}{l}\begin{array}{l}\text { Registros identificados através das bases de dados } \\
\text { (Lilacs) }\end{array} \\
\underline{1770 \text { artigos }}\end{array}$ \\
\hline \multicolumn{2}{|c|}{ SELEÇÃO } \\
\hline \multicolumn{2}{|c|}{$\begin{array}{l}\text { Artigos originais e disponíveis na integra, entre os anos de } 2015 \text { a 2019, no idioma português e artigos } \\
\text { observacionais citáveis desenvolvidos em território nacional. }\end{array}$} \\
\hline \multicolumn{2}{|c|}{ CRITERIOS DE EXCLUSÃO } \\
\hline \multicolumn{2}{|c|}{$\begin{array}{l}\text { Artigos de revisão sistemática e bibliográfica, estudos duplicados, estudos que não estejam relacionados ao tema } \\
\text { principal da pesquisa e. }\end{array}$} \\
\hline \multicolumn{2}{|c|}{$\overline{\text { ELEGIBILIDADE }}$} \\
\hline Estudo com dados de prevalência de & ade física em adolescentes escolares. \\
\hline
\end{tabular}

Fluxograma 1: Processo de triagem dos estudos da revisão sistemática. 
Tabela 2: Síntese dos estudos acerca de prevalência de inatividade física em crianças e adolescentes.

\begin{tabular}{|c|c|c|c|c|c|c|c|}
\hline Autores/Ano & Local & $\begin{array}{ll}\text { Tipo } & \text { de } \\
\text { estudo } & \\
\end{array}$ & Seleção & Amostra & $\begin{array}{l}\text { Faixa } \\
\text { etária }\end{array}$ & Instrumentos & $\begin{array}{l}\text { Prevalência de } \\
\text { inatividade física }\end{array}$ \\
\hline $\begin{array}{l}\text { Alberto et al. } \\
(2015)\end{array}$ & Macapá & Transversal & Conglomerado & 1.060 & $\begin{array}{l}10 \text { a } 15 \\
\text { anos }\end{array}$ & IPAQ - curto ${ }^{1}$ & $69,5 \%$ \\
\hline $\begin{array}{l}\text { Alberto et al. } \\
(2016)\end{array}$ & Macapá & Transversal & Conglomerado & 1.060 & $\begin{array}{l}10 \text { a } 15 \\
\text { anos }\end{array}$ & IPAQ - curto ${ }^{1}$ & $69,5 \%$ \\
\hline $\begin{array}{l}\text { Bezerra et al. } \\
(2015)\end{array}$ & Pernambuco & Transversal & Aleatória & 4.207 & $\begin{array}{l}14 \text { a } 19 \\
\text { anos }\end{array}$ & $\mathrm{GSHS}^{2}$ & $38,2 \%$ \\
\hline $\begin{array}{l}\text { Cureau et al. } \\
(2016)\end{array}$ & Brasil & Transversal & Equiprobabilidade & 74.589 & $\begin{array}{l}12 \text { a } 17 \\
\text { anos }\end{array}$ & SAPAC20 3 & $54,3 \%$ \\
\hline $\begin{array}{l}\text { Ferreira et al. } \\
(2016)\end{array}$ & Pelotas & Transversal & Sorteio & 8.661 & $\begin{array}{l}12 \text { a } 16 \\
\text { anos }\end{array}$ & Helena & $69,2 \%$ \\
\hline $\begin{array}{l}\text { Fonseca et al. } \\
\text { (2019) }\end{array}$ & $\begin{array}{l}\text { Montes } \\
\text { Claros }\end{array}$ & Transversal & Conglomerado & 633 & $\begin{array}{l}10 \text { a } 16 \\
\text { anos }\end{array}$ & IPAQ - curto ${ }^{1}$ & $60 \%$ \\
\hline $\begin{array}{l}\text { Guedes et al. } \\
\text { (2019) }\end{array}$ & Nordeste & Transversal & Conveniência & 158 & $\begin{array}{l}11 \text { a } 19 \\
\text { anos }\end{array}$ & NANDA & $68,3 \%$. \\
\hline $\begin{array}{l}\text { Guilherme et al. } \\
\text { (2015) }\end{array}$ & Paranavaí & Transversal & Aleatória & 566 & $\begin{array}{l}10 \text { a } 14 \\
\text { anos }\end{array}$ & IBGE & $56,1 \%$ \\
\hline $\begin{array}{l}\text { Lourenço et al. } \\
\text { (2018) }\end{array}$ & Uberaba & Transversal & Conglomerado & 1.009 & $\begin{array}{l}14 \text { a } 19 \\
\text { anos }\end{array}$ & QCAC $^{4}$ & $81,6 \%$ \\
\hline $\begin{array}{l}\text { Menezes et al. } \\
(2015)\end{array}$ & Sergipe & Transversal & Conglomerado & 3.992 & $\begin{array}{l}14 \text { a } 19 \\
\text { anos }\end{array}$ & $\mathrm{GSHS}^{2}$ & $77,5 \%$ \\
\hline $\begin{array}{l}\text { Santana et al. } \\
(2017)\end{array}$ & Pernambuco & Transversal & Conglomerado & 6.039 & $\begin{array}{l}14 \text { a } 19 \\
\text { anos }\end{array}$ & $\mathrm{GSHS}^{2}$ & $79,3 \%$ \\
\hline $\begin{array}{l}\text { Tanaka et al. } \\
(2015)\end{array}$ & São Paulo & Transversal & Aleatório & 91 & $\begin{array}{l}10 \text { a } 19 \\
\text { anos }\end{array}$ & FLORINDO & $71,4 \%$ \\
\hline $\begin{array}{l}\text { Vitorino et al. } \\
(2015)\end{array}$ & Goiânia & Transversal & Sorteio & 132 & $\begin{array}{l}14 \text { a } 18 \\
\text { anos }\end{array}$ & IPAQ - curto ${ }^{1}$ & $19,7 \%$ \\
\hline
\end{tabular}

Legenda: ${ }^{1}$ Versão curta do questionário internacional de atividade física; ${ }^{2}$ Global School-based Student Health Survey; ${ }^{3}$ Self-Administered Physical Activity Checklist20; ${ }^{4}$ Questionário de comportamento do adolescente catarinense.

\section{RESULTADOS E DISCUSSÃO}

Na Tabela 2, apresenta-se a síntese dos resultados de estudos realizados em diversas cidades do Brasil, no período de 2015 a 2019, de forma a contribuir com informações relevantes ao tema. A maior proporção dos estudos selecionados foi realizada nas Regiões Nordeste e Sudeste. Trata-se de estudos de delineamento transversal, realizados com amostras que variaram de 91 a 74.589 adolescentes com idade entre 10 e 19 anos.

Tanaka et al. (2015) avaliaram em seu estudo 91 adolescentes com idade entre 10 e 19 anos no estado de São Paulo com o intuito de verificar a prevalência da inatividade física, empregou como parâmetro o questionário proposto por Florindo. Foram considerados inativos aqueles que praticavam um tempo abaixa de 300 minutos de exercício físico semanal, em seus resultados observaram o sexo feminino com maior prevalência em relação ao sexo masculino ( $80 \%$ e $61 \%$ respectivamente).

Santana et al. (2017) em seu estudo avaliaram 6.039 adolescentes, com idade entre 14 e 19 anos do estado de Pernambuco, com relação a coleta de informações, foi usado o questionário Global School-based Student Health Survey (GSSH), depois da coleta de dados, foi identificado uma prevalência de inatividade física maior nos meninos $(81,6 \%)$ quando comparados as meninas $(77,8 \%)$. Os autores ainda relatam que os adolescentes inativos apresentam uma pressão arterial elevada quando comparados a adolescentes ativos.

Menezes et al. (2015) usaram o questionário Global School-based Student Health Survey (GSSH), assim como (SANTANA et al., 2017), para avaliar o nível de atividade física, em seu estudo participaram 3.992 
adolescentes do ensino médio da rede estadual de Sergipe, com idade entre 14 e 19 anos, a rede estadual foi escolhido, por conter cerca de $80 \%$ dos escolares do estado, onde a prevalência geral foi semelhante ao estudo de (SANTANA et al., 2017) 77,5\%, porém em relação ao sexo as prevalências se contrariaram, onde neste o sexo feminino obtiveram maior prevalência $86 \%$, quando comparada com o sexo masculino com $71,0 \%$. Ressaltando ainda que a inatividade física foi mais prevalente em adolescentes residentes na zona urbana.

Bezerra et al. (2015) usaram no seu estudo o questionário Global School-based Student Health Survey (GSSH) assim como nos estudos de (MENEZES et al., 2015; SANTANA et al., 2017) para avaliar a inatividade física de 4.207 estudantes, com variação de idade entre 14 e 19 anos, escolares da rede estadual de Pernambuco. Após análise de dados o resultado foi de $38,2 \%$ de estudantes inativos, considerado inferior aos resultados encontrados nos estudos de (MENEZES et al., 2015; SANTANA et al., 2017). Em comparação ao sexo, a prevalência foi evidenciada em maior proporção entre estudantes do sexo feminino $48,9 \%$ em relação aos do sexo masculino $22,5 \%$.

Guilherme et al. (2015) estudaram 566 escolares, com idade de 10 a 14 anos, da rede pública e privada da cidade de Paranavaí, o instrumento utilizado para examinar o nível de atividade física foi o questionário proposto pelo IBGE, onde se considerou inativo fisicamente os adolescentes com uma frequência de pratica de atividade física menor que 300 minutos semanais, em seus resultados, observaram que em relação ao sexo, a maior prevalência foi encontrada no sexo masculino 53,3\% de adolescentes inativos e que essa prevalência se mantem elevada em adolescentes com excesso de peso corporal quando comparados a adolescentes com peso adequado.

Cureau et al. (2016) realizaram um estudo com 74.589 adolescentes, com idades de 12 e 17 anos em todo o território nacional, para indicar o nível de atividade física dos adolescentes, foi empregada uma adequação do Self-Administered Physical Activity Checklist, onde o mesmo consegue determinar a frequência e o tempo em que os adolescentes praticaram alguma atividade física. Foram incluídos como inativos os adolescentes brasileiros que não acumularam pelos menos 300 min por semana de atividade física, em nível nacional a maior prevalência foi no sexo feminino $70,7 \%$ comparada a adolescentes do sexo masculino 38,0\%, onde as maiores prevalências de inatividade física estiveram nas regiões Nordeste e Sudeste no sexo feminino e na região Sul entre os adolescentes do sexo masculino.

Ferreira et al. (2016) utilizaram no seu estudo, uma amostra composta por 8.661 escolares da cidade de Pelotas, com idades de 12 a 16 anos, para investigação do nível de atividade física, foi empregado o instrumento HELENA, para constatar o tempo em relação a pratica de atividade física, após a coleta de dados, foi verificado a prevalência total de inatividade física de $69,2 \%$ nos adolescentes.

Vitorino et al. (2015) avaliaram 132 adolescentes, com idades de 14 a 18 anos, das escolas da rede estadual de Goiânia, utilizando o questionário IPAQ, para verificar o tempo gasto com pratica de atividade física moderada e vigorosa, e com isso, foi identificado a prevalência de inatividade física em $19,7 \%$ dos adolescentes, o estudo não mencionou qual a prevalência em relação ao sexo.

Alberto et al. (2015) usaram o IPAQ na sua versão curta, para avaliar 1.060 adolescentes escolares 
com idade de 10 a 15 anos da cidade Macapá, com a finalidade de identificar a prevalência de inatividade física, assim como (VITORINO et al., 2015). Em contrapartida ao estudo de (VITORINO et al., 2015) a prevalência foi elevada chegando a $69,5 \%$, sendo mais pronunciado entre as meninas com $70,3 \%$ em relação aos rapazes com 67,8\%, demonstrando que 7 de cada 10 adolescentes entre 10 e 15 anos, de ambos os sexos não praticam atividade física em níveis adequados.

Alberto et al. (2016) também utilizaram o IPAQ na sua versão curta, como parâmetro para avaliação de 1.060 adolescentes com idade de 10 a 15 anos da cidade de Macapá, os resultados demonstram níveis altos de inatividade física entre os adolescentes avaliados, onde foi encontrada uma prevalência de inatividade física para ambos os sexos $69,5 \%$. A prevalência foi parecida em ambos os sexos, porem demonstra uma pequena elevação em relação ao sexo feminino, destacando que os adolescentes com idade de 12 e 13 anos, de ambos os sexos foram considerados mais inativos.

Fonseca et al. (2019) o público alvo da análise foi constituída por 633 adolescentes escolares de 10 a 16 anos, matriculados na rede pública de ensino da cidade de Montes Claros, o instrumento utilizando para identificar a inatividade física na pesquisa foi o questionário IPAQ na sua versão curto assim como nos estudos de (ALBERTO et al., 2015; VITORINO, 2015; ALBERTO et al., 2016) onde após a avaliação dos questionários, verificou-se uma prevalência de $60 \%$ em ambos os sexos, assemelhando-se aos resultados encontrados por (ALBERTO et al., 2015; ALBERTO et al., 2016) e contrariando os resultados obtido por (VITORINO, 2015).

Lourenço et al. (2018) realizaram o seu estudo com 1.009 adolescentes, de 14 a 19 anos das escolas públicas e privadas da cidade de Uberaba, foi utilizado o questionário de comportamento do adolescente catarinense para colher informações para o estudo, onde se pode observa que 8 em cada 10 adolescentes são inativos fisicamente, sendo esta prevalência mais elevada no sexo masculino $84,9 \%$, os autores afirmam ainda que a série de estudo, situações como estagio ou trabalho estão diretamente relacionada a alta prevalência.

Guedes et al. (2019) realizaram seu estudo na região nordeste do Brasil, com 158 adolescentes, cuja idade varia de 11 a 19 anos, utilizando o formulário NANDA, para medir o nível de atividade física dos estudantes, onde foi encontrado o valor para inatividade física de 68,3\%, o estudo não demonstrou a prevalência em relação ao sexo, porém afirma que um dos principais fatores para a alta prevalência de inatividade encontrada no estudo foi o conhecimento deficiente dos adolescentes sobre os benefícios que a atividade física traz à saúde.

A pratica de atividade é essencial para manutenção da saúde e qualidade de vida, e a falta da mesma pode ocasionar algumas patologias associadas à inatividade física, assim (OLIVEIRA et al., 2015) destacam em seu estudo a inatividade física como um dos problemas de saúde pública na atualidade, por conta de sua associação com as doenças crônicas não transmissíveis, como: diabetes, doenças respiratórias crônicas, cardiovasculares e câncer.

No Brasil, (BRASIL, 2014) em seu último levantamento, verificou que a prevalência de inatividade física vem se elevando, atingindo $47,5 \%$ da população em 2015. Com isso, se a atividade física for oferecida 
durante a infância e adolescência, terá grande importância na construção da pratica de atividade física no decorrer da vida, tornando as crianças e adolescentes mais ativos, e assim podendo levar esse comportamento para vida adulta.

De acordo com essas ocorrências, existe uma inquietação em relação à quantidade mínima necessária de atividade física capaz de incidir nos benefícios a saúde dos adolescentes, onde segundo a (OMS, 2010) recomenda que os adolescentes devam praticar atividade física de intensidade moderada a vigorosa, por pelo menos 60 minutos diário, acumulando 300 minutos semanais. Cureau et al. (2016) relataram no seu estudo que existem uma série de pesquisas que utilizam a recomendação da pratica de atividade física por pelo menos 300 minutos semanais, porém ainda trazem que já existem evidencias que volumes menores podem beneficiar o indivíduo.

Dorneles et al. (2019) identificaram no seu estudo que a pratica regular de atividade física, promove alterações benéficas ao organismo, causando efeitos centrais como: controle de ansiedade e controle emocional, além de efeitos periféricos como: a diminuição de dores. Relatam ainda que a atividade física tem efeitos positivos na qualidade do sono, humor e bem-estar físico e mental. Silva et al. (2018) retrataram que a atividade física é utilizada para a valorização da promoção da saúde, ajudando principalmente na vigilância e controle de doenças cardiovasculares, perca de peso excessiva, e síndromes metabólicas, como forma de firma a pratica de hábitos saudáveis.

Diante dos dados apresentados nessa revisão, pode se enfatizado que a inatividade física é uma preocupação nacional, a sua prevalência, por mais que tenha variação de acordo com alguns estudos, é bastante significativa. Com esses dados tão elevados se faz necessário à intervenção dos profissionais de saúde, juntamente com a escola, para tentar fazer com que se obtenha uma redução desse problema.

\section{CONCLUSÕES}

Com base nos dados coletados nesse estudo, pode-se concluir que todos utilizam corte transversal e que a metodologia aplicada para verificar a prevalência de inatividade física, são as recomendações da OMS, mudando apenas o instrumento utilizado para verificação dos dados, verifica-se também uma alta prevalência de inatividade física em ambos os sexos, sendo mais prevalente no sexo feminino, com isso é preciso que a prática de atividade física seja estimulada desde a infância, reduzindo esses dados e por consequência a diminuição de futuras patologias causadas pela inatividade física.

Esses dados podem ajudar no desenvolvimento de futuras pesquisas, e elaboração de políticas públicas voltada para a promoção da atividade física no âmbito escolar, com a intenção de modificar de alguma forma esses dados tão elevados de inatividade física, tendo em vista, o melhoramento da saúde das crianças e adolescentes.

Destaca-se como limitação da presente pesquisa a dificuldades relacionadas aos estudos para essa revisão foi o período de publicação de cada artigo, utilizando estudos publicados apenas nos últimos cinco anos, a ponte de corte e os instrumentos utilizado para realização de cada pesquisa, onde falta de padronização de um único questionário, pode influenciar no resultado, ou até mesmo a falta de compromisso 
do aluno ao respondê-los.

Novos estudos devem analisar qual a influência que a inatividade física tem sobre os adolescentes, relacionando a diversas variáveis, tais como: o lugar que mora, a renda familiar, a relação da pratica na escola. Outro ponto importante seria a padronização de um questionário especifico para avaliar a inatividade física, onde as variabilidades dos resultados possam esta atribuída aos diferentes instrumentos utilizados, tendo assim resultados mais fidedignos.

\section{REFERÊNCIAS}

ALBERTO, A. A. D.; FIGUEIRA JUNIOR, A. J.. Prevalência de inatividade física e sua associação com variáveis sociodemográficas em adolescentes do Município de Macapá/AP. Revista Brasileira Ciência e Movimento, v.23, n.4, p.80-93, 2015.

ALBERTO, A. A. D.; FIGUEIRA JUNIOR, A. J.. Prevalência de inatividade física em adolescentes e sua associação com variáveis socioculturais. Pensar A Prática, v.19, n.4, p.801813, 2016. DOI: http://dx.doi.org/10.5216/rpp.v19i4.35317

BEZERRA, J.; LOPES, A. S.; HARDMAM, C. M.; TASSITANO, R. M.; TENÓRIO, M. C. M.; BARROS, M. V. G.. Consumo de bebidas alcoólicas e tabagismo: associação com inatividade física no lazer e comportamento sedentário. Revista Andaluza de Medicina Del Deporte, v.8, n.1, p.1-6, 2015. DOI: http://dx.doi.org/10.1016/j.ramd.2014.08.002

BRASIL. Ministério da Saúde. Vigitel Brasil 2014: vigilância de fatores de risco e proteção para doenças crônicas por inquérito telefônico. Brasília: Ministério da Saúde, 2014.

CUREAU, F. V.; SILVA, T. L. N.; BLOCH, K. V.; FUJIMORI, E.; BELFORT, D. R.; CARVALHO, K. M. B.; LEON, E. B.; VASCONCELLOS, M. T. L.; EKELUND, U. L. F.; SCHAAN, B. D. E.. Leisure-time physical inactivity in Brazilian adolescents. Revista de Saúde Pública, v.50, n.1, p.1-11, 2016. DOI: http://dx.doi.org/10.1590/s01518$\underline{8787.2016050006683}$

DORNELES, R.; BERGMANN, G.; SILVA, M.. Risk for exercise dependence in health clubs. Psicologia, Saúde \& Doença, v.20, n.1, p.137-148, 2019. DOI: http://dx.doi.org/10.15309/19psd200111

FERREIRA, R. W.; ROMBALDI, A. J.; RICARDO, L. I. C.; HALLAL, P. C.; AZEVEDO, M. R.. Prevalence of sedentary behavior and its correlates among primary and secondary school students. Revista Paulista de Pediatria, v.34, n.1, p.56-63, 2016. DOI: http://dx.doi.org/10.1016/j.rppede.2015.09.002

FONSECA, A. D. G.; CUNHA, F. O.; BARBOSA, I. A.; SILVA, J. O.; ARAÚJO, D. D.; SILVA, C. S. O.. Quality of life in adolescents related to sex, family income and physical activity. Reme Revista Mineira de Enfermagem, v.23, p.1-7, 2019. DOI: http://dx.doi.org/10.5935/1415-2762.20190093

GUEDES, N. G.; LOPES, M. V. O.; SILVA, V. M.; FARIAS, L. J. R.; SILVA, L. A.; BESSA, C. C.. Clinical validation of factors associated with sedentary lifestyle in adolescents. Revista Rene, v.20, p.1-8, 2019. DOI:

http://dx.doi.org/10.15253/2175-6783.20192040395
GUILHERME, F. R.; MOLENA-FERNANDES, C. A.; GUILHERME, V. R.; FÁVERO, M. T. M.; REIS, E. J. B.; RINALDI, W..

Inatividade física e medidas antropométricas em escolares de Paranavaí, Paraná, Brasil. Revista Paulista de Pediatria, v.33, n.1, p.50-55, 2015. DOI:

http://dx.doi.org/10.1016/j.rpped.2014.11.009

IBGE. Instituto Brasileiro de Geografia e Estatística. Pesquisa Nacional de Saúde do Escolar, 2015. Rio de Janeiro: IBGE; 2015.

LOURENÇO, C. L. M.; ZANETTI, H. R.; AMORIM, P. R. S.; MOTA, J. A. P. S.; MENDES, E. L.. Comportamento sedentário em adolescentes: prevalência e fatores associados. Revista. Brasileira Ciência e Movimento, v.26, n.3, p.23-32, 2018.

MARQUES, A.; CALMEIRO, L.; LOUREIRO, N.; RASQUILHO, D.; MATASO, M. G.. Health complaints among adolescents: Associations with more screen-based behaviours and less physical activity. Journal of Adolescence, v.44, p.150-157, 2015. DOI:

http://dx.doi.org/10.1016/j.adolescence.2015.07.018

MENEZES, A. S.; DUARTE, M. F. S.. Condições de vida, inatividade física e conduta sedentária de jovens nas áreas urbana e rural. Revista Brasileira de Medicina do Esporte, v.21, n.5, p.338-344, 2015. Dol: http://dx.doi.org/10.1590/1517-869220152105145322

OLIVEIRA, M. M.; CAMPOS, M. O.; ANDREAZZI, M. A. R.; MALTA, D. C.. Características da Pesquisa Nacional de Saúde do Escolar - PeNSE. Epidemiologia e Serviços de Saúde, v.26, n.3, p.605-616, 2017. DOI: http://dx.doi.org/10.5123/s167949742017000300017

OLIVEIRA, M. R.; SILVA, V. M.; GUEDES, N. G.; LOPES, M. V. O.. Clinical Validation of the 'Sedentary Lifestyle' Nursing Diagnosis in Secondary School Students. The Journal of School Nursing, v.32, n.3, p.186-194, 2015. DOI: http://dx.doi.org/10.1177/1059840515588956

OMS. Organização Mundial de Saúde. Physicalactivity. OMS, 2018.

SANTANA, F. S.; PALMEIRA, A. C.; SANTOS, M. A. M.; FARAH, B. Q.; SOUZA, B. C. C.; RITTI-DIAS, R. M.. Association between active commuting and elevated blood pressure in adolescents. Einstein, São Paulo, v.15, n.4, p.415-420, 2017. DOI: http://dx.doi.org/10.1590/s1679-45082017ao4093

SILVA, J.; ANDRADE, A.; CAPISTRANO, R.; LISBOA, T.; ANDRADE, R. D.; FELDEN, É. P. G.; BELTRAME, T. S.. Níveis insuficientes de atividade física de adolescentes associados a 
fatores sociodemográficos, ambientais e escolares. Ciência \& Saúde Coletiva, v.23, n.12, p.4277-4288, 2018. DOI: http://dx.doi.org/10.1590/1413-812320182312.30712016

SILVA, K. V. L. G.; GONÇALVES, G. A. A.; SANTOS, S. B.; MACHADO, M. F. A. S.; REBOUÇAS, C. B. A.; SILVA, V. M.; XIMENES, L. B.. Training of adolescent multipliers from the perspective of health promotion core competencies. Revista Brasileira de Enfermagem, v.71, n.1, p.89-96, 2018. DOI: http://dx.doi.org/10.1590/0034-7167-2016-0532

TANAKA, L. F.; LATORRE, M. R. D. O.; SILVA, A. M.; KONSTANTYNER, T. C. R. O.; PERES, S. V.; MARQUES, H. H. S. Alta prevalência de sedentarismo em adolescentes que vivem com HIV/Aids. Revista Paulista de Pediatria, v.33, n.3, p.326-331, 2015. DOI:

http://dx.doi.org/10.1016/i.rpped.2014.12.003

VITORINO, P. V. O.; BARBOSA, M. A.; SOUSA, A. L. L.; JARDIM, P. C. B. V.; FERREIRA, S. S.. Prevalência de estilo de vida sedentário entre adolescentes. Acta Paulista de Enfermagem, v.28, n.2, p.166-171, 2015. DOI: http://dx.doi.org/10.1590/1982-0194201500028

WARBURTON, D.; BREDIN, S. S. D.. Health benefitsofphysicalactivity. Current Opinion In Cardiology, v.32, n.5, p.541-556, 2017. DOI:

http://dx.doi.org/10.1097/hco.0000000000000437

A CBPC - Companhia Brasileira de Produção Científica (CNPJ: 11.221.422/0001-03) detém os direitos materiais desta publicação. Os direitos referem-se à publicação do trabalho em qualquer parte do mundo, incluindo os direitos às renovações, expansões e disseminações da contribuição, bem como outros direitos subsidiários. Todos os trabalhos publicados eletronicamente poderão posteriormente ser publicados em coletâneas impressas sob coordenação da Sapientiae Publishing, da Companhia Brasileira de Produção Científica e seus parceiros autorizados. Os (as) autores (as) preservam os direitos autorais, mas não têm permissão para a publicação da contribuição em outro meio, impresso ou digital, em português ou em tradução. 